\title{
O Uso de Dados de Alta Freqüência na Estimação da Volatilidade e do Valor em Risco para o IBOVESPA*
}

\author{
João Maurício de Souza Moreira** \\ Eduardo Facó Lemgruber***
}

Sumário: 1. Introdução; 2. Metodologia e amostra; 3. Resultados; 4. Conclusão.

Este artigo investiga o uso de dados de alta freqüência na estimação das volatilidades diária e intradiária do IBOVESPA e no cálculo do valor em risco (VaR). Os modelos GARCH e EGARCH são usados em conjunto com métodos determinísticos de filtragem de sazonalidade para a previsão da volatilidade e do VaR intradiários. Uma comparação com o método não-paramétrico baseado no quantil empírico é efetuada. No cálculo do VaR diário, dois métodos simples de previsão buscam captar a informação de volatilidade contida nos dados de alta freqüência. O primeiro utiliza o desvio padrão amostral com janela móvel e o segundo faz uso da técnica de alisamento exponencial. Alguns métodos tradicionais aplicados a dados diários são usados para comparação. No cálculo do VaR diário, os dois métodos baseados em dados intradiários apresentaram bom desempenho. No cálculo do VaR intradiário, os resultados mostram que a filtragem do padrão sazonal é indispensável à obtenção de medidas úteis de volatilidades com o uso dos modelos GARCH e EGARCH.

This paper investigates the use of high frequency data in the estimation of daily and intraday volatility, in order to compute value at risk (VaR) forecasts for the IBOVESPA. GARCH models and deterministic methods for the filtering of seasonal patterns are used in the computation of intraday volatility and VaR forecasts. A comparison with a non-parametric method based on the empirical quantile is done. For daily VaR two simple methods seek to extract the volatility information conveyed by the high frequency data. The first method is based on the sample standard deviation with a moving window, while the second is based on exponencially weighted moving average. Some traditional methods applied to daily data are used for benchmarking. Both methods tested

\footnotetext{
${ }^{*}$ Artigo recebido em dez. 2002 e aprovado em mai. 2003. Os autores agradecem à Bolsa de Valores de São Paulo pelos dados utilizados neste trabalho.

${ }^{* *}$ Pesquisador do Departamento de Estudos e Pesquisas do Banco Central do Brasil.

${ }^{* * *}$ Professor do COPPEAD/UFRJ.
} 
present good performance. For intraday VaR the results indicate that the filtering of the seasonal pattern is a fundamental step in obtaining useful forecasts of volatility and VaR.

\section{Introdução}

Tradicionalmente o cálculo de estimativas da volatilidade de retornos financeiros, bem como a sua aplicação à determinação do valor em risco (VaR), tomam como base as variações diárias nos preços dos ativos (Goodhart e O'hara, 1997). Contudo, a crescente disponibilidade de dados registrados em intervalos de tempo cada vez mais curtos, somada aos avanços tecnológicos na área de computação, abriram espaço para a investigação empírica voltada para a análise e aplicação dos dados de alta freqüência em uma diversidade de aspectos dos mercados financeiros. A possibilidade de se obter estimativas mais precisas de volatilidade tem despertado o interesse de muitos pesquisadores. ${ }^{1}$ Um benefício prático imediato seria o cômputo de valores em risco que representem com maior acurácia as possibilidades de perda. Não só o tradicional VaR diário pode ser beneficiado pela informação adicional contida nos dados de alta freqüência, como também há a possibilidade de se obter um VaR intradiário voltado para operações freqüentes em mercados de alta liquidez.

Andersen e Bollerslev (1998b,a, 1999) concluem que a explícita incorporação da informação contida em dados de alta freqüência melhora significativamente as projeções da volatilidade dos retornos diários, tanto na teoria como na prática. Taylor e Xu (1997) utilizam uma série de retornos do marco alemão em relação ao dólar americano, registrados a cada cinco minutos, para avaliar a existência de informação que seja incremental àquela obtida implicitamente a partir de opções cambiais. Os resultados corroboram esta possibilidade. Goodhart e O'hara (1997) lembram que os retornos intradiários apresentam comportamento particular sistemático, cujo reconhecimento é fundamental para a modelagem da volatilidade. Usualmente identificado em mercados financeiros onde a negociação não é viável antes da abertura formal do pregão, o padrão sazonal consiste em valores máximos para a volatilidade na abertura, queda rápida para valores mínimos no meio do

\footnotetext{
${ }^{1} \mathrm{O}$ interesse no uso de dados de alta freqüência para o estudo da volatilidade em séries financeiras tem se revelado mais intensamente nos últimos anos. Contudo, o método do valor extremo de Parkinson (1980) já buscava mostrar que a estimação da volatilidade, relativa a séries de retornos de ações, efetuada com base nas cotações máxima e mínima registradas a cada dia é superior àquela computada somente a partir dos preços de fechamento.
} 
dia, voltando a crescer até o fechamento. ${ }^{2}$ Entretanto, Andersen e Bollerslev (1997) alertam que os padrões sistemáticos observados em séries financeiras de alta freqüência, em mercados de ações e de câmbio, são ignorados em boa parte da literatura empírica de microestrutura de mercado, o que explicaria a aparente inadequação dos modelos tradicionais de volatilidade quando aplicados a séries de alta freqüência. Os autores mostram que a estimação e filtragem do componente periódico intradiário da volatilidade é factível e sua explícita incorporação na especificação dos modelos usados permite eliminar a maior parte das distorções atribuídas ao comportamento sazonal.

A modelagem do VaR é uma aplicação natural e imediata dos modelos de volatilidade. No entanto, apesar dos inúmeros estudos acerca da volatilidade de dados intradiários, pouco se tem escrito sobre a sua aplicação ao cálculo de VaR. Beltratti e Morana (1999) avaliam o uso de dados de alta freqüência para a obtenção de medidas de risco. Em particular, os autores buscam avaliar se os resultados obtidos na análise da volatilidade com dados intradiários trazem implicações no cálculo da volatilidade para períodos múltiplos de um dia. Giot (2000b) usa uma série de cotações de alta freqüência de ações da IBM negociadas na bolsa de Nova Iorque em conjunto com modelos GARCH e EGARCH para caracterizar a volatilidade intradiária, levando em conta a existência de padrões sazonais intradiários. Os modelos são usados para prever o VaR de $5 \%$ para os intervalos de 10 e 15 minutos seguintes, admitindo distribuição normal dos retornos filtrados. Na mesma linha, Giot (2000a) analisa amplos movimentos de preços intradiários através da aferição do desempenho de diversos modelos paramétricos e não-paramétricos de volatilidade, incluindo os da família GARCH, o RiskMetrics e os baseados no quantil empírico e na distribuição de Pareto, na projeção do VaR intradiário. Em outra vertente, Giot e Laurent (2001a) desenvolvem um modelo para computar o VaR diário a partir do uso de dados intradiários. Mais especificamente, usam a volatilidade realizada diária, dada pela soma dos quadrados dos retornos intradiários ao longo de um dia, como uma medida de volatilidade diária agregada para duas séries de retornos de índices de ações registrados a cada 15 minutos.

O objetivo deste trabalho é avaliar o uso de dados de alta freqüência na previsão de volatilidade e cálculo do VaR para os horizontes diário e intradiário, a partir da série de cotações do IBOVESPA registradas a cada 15 minutos, entre abril de 1998 e julho de 2001. Em uma primeira etapa, são avaliadas estimati-

\footnotetext{
${ }^{2} \mathrm{O}$ mesmo padrão sazonal se verifica para o volume de negociações e para o spread entre as cotações de compra e venda dos ativos. Alguns estudos de microestrutura de mercado buscam explicar o comportamento da volatilidade intradiária com base na correlação entre as três variáveis (ver Goodhart e O'hara (1997)).
} 
vas de VaR intradiário calculadas com base nos modelos de volatilidade GARCH e EGARCH. As distorções sugeridas pela literatura, decorrentes do fator sazonal intradiário, bem como o efeito da aplicação de procedimentos de filtragem de sazonalidade, são evidenciados. Na segunda parte do trabalho são aferidas as estimativas de VaR diário calculadas por métodos de estimação de volatilidade que incorporam informações contidas nos retornos intradiários. Os resultados no âmbito intradiário indicam que o uso de dados de alta frequiência com modelos da família GARCH gera medidas confiáveis do VaR para os próximos 15 minutos. No horizonte diário, o ótimo desempenho dos métodos de VaR avaliados, igual ou superior ao de métodos tradicionais baseados em dados diários, mostra que os dados intradiários podem trazer melhoras significativas para o VaR de um dia.

Este artigo se desenvolve com a seção 2 descrevendo os dados e a metodologia empregada em seu tratamento, na investigação estatística, na estimação dos parâmetros dos modelos de previsão de volatilidade e na avaliação dos resultados. A seção 3 apresenta os resultados obtidos e a seção 4 reúne as conclusões e observações finais.

\section{Metodologia e Amostra}

Dados de alta freqüência registrados de forma quase contínua demandam modelos que tratem observações com diferentes intervalos de tempo entre si. A organização dos dados em intervalos fixos de tempo permite o uso de modelos econométricos tradicionais. O intervalo entre as observações deve ser pequeno o bastante para dar uma idéia precisa da volatilidade intradiária, mas não tão pequeno a ponto de gerar muitas observações contíguas idênticas, onde nenhuma nova informação é transmitida. Giot (2000a) considera ótimo o intervalo de 15 minutos, enquanto Andersen (2000) acredita que a freqüência de 5 minutos seria, aproximadamente, a mais alta para a qual as propriedades da série não seriam distorcidas por aspectos da microestrutura de mercado. Para este estudo, a série utilizada é composta de cotações do Índice da Bolsa de Valores de São Paulo, IBOVESPA, registrados a cada 15 minutos durante o horário de pregão no período de 6/4/1998 a 19/7/2001, correspondendo a 803 dias úteis e 23.287 observações intradiárias.

Em um dia padrão, os dados encontram-se organizados em blocos diários de 29 cotações, equivalendo a 28 intervalos de 15 minutos, não importando o horário específico em que ocorreram, mas sim a sua ordem cronológica, com foco nas 29 últimas cotações de cada dia. No banco de dados original, o número de cotações varia entre 29 e 33 por dia. Com o objetivo de homogeneizar a distribuição das 
observações, optou-se por excluir todas as observações relativas a dias de expediente reduzido e às primeiras cotações dos dias em que o número de observações excede 29. ${ }^{3}$ Este procedimento levou em conta que a maior parte do período em estudo já apresenta originalmente 29 observações diárias.

A amostra é integralmente utilizada na investigação dos fatos estilizados das séries de retornos e na aferição das projeções de VaR diário com base em dados intradiários. Para a avaliação do VaR intradiário usou-se apenas uma parte destes dados, relativa ao período de 3/4/2000 a 6/10/2000, totalizando 130 dias úteis e 3.770 cotações intradiárias. Neste período o pregão apresentou horário e duração constantes, com 29 cotações a cada dia, não sendo necessário qualquer ajuste. O padrão sazonal intradiário seria impropriamente avaliado se fossem desconsiderados os primeiros retornos registrados no dia. Daqui em diante, o período de 6/4/1998 a 19/7/2001 será referido como amostra completa e o período de $3 / 4 / 2000$ a $6 / 10 / 2000$ como amostra reduzida.

De acordo com o procedimento usual, são utilizados os retornos logarítmicos em toda a nossa análise, retomando-se os retornos efetivos por ocasião da aferição das projeções de VaR. Para a amostra completa, seja $P_{t, 0}$ a primeira cotação do IBOVESPA no dia $t$ (abertura) e seja $R_{t, j}$ o retorno relativo ao $j$-ésimo intervalo de quinze minutos do dia $t$, onde $t=1,2,3, \ldots, 802$ e $j=1,2,3, \ldots, 28$. Para o cálculo do primeiro retorno no dia $t$, tem-se $R_{t, 1}=\ln \left(P_{t, 1} / P_{t-1,28}\right) .{ }^{4}$ Para os demais retornos intradiários, tem-se $R_{t, j}=\ln \left(P_{t, j} / P_{t, j-1}\right)$. E para cada dia, onde $P_{t}=P_{t, 28}$ é a cotação de fechamento em $t$ e $R_{t}=\ln \left(P_{t} / P_{t-1}\right)$ é o retorno diário relativo ao dia $t$, tem-se $R_{t}=\sum R_{t, j}$. Vale observar que este cálculo para o primeiro retorno incorpora as informações relativas ao intervalo entre o fechamento do pregão do dia anterior e a abertura do pregão do dia em questão (overnight). ${ }^{5}$ $\mathrm{Na}$ amostra reduzida, destinada à avaliação do VaR intradiário, não há a necessidade de incorporar o overnight no cálculo do primeiro retorno, uma vez que se está apenas interessado no comportamento intradiário da volatilidade. Neste caso, tem-se $R_{t, 1}=\ln \left(P_{t, 1} / P_{t, 0}\right)$. Os demais retornos são calculados de forma idêntica à especificada para a amostra completa.

\footnotetext{
${ }^{3}$ Dias com menos de 29 observações ocorreram em datas especiais como Quarta-feira de Cinzas e Vésperas de Natal e Ano Novo, assim como em consequëncia de falhas no suprimento de energia elétrica.

${ }^{4} \mathrm{Ou}$ seja, a primeira cotação do dia é desprezada, conforme procedimento adotado por Giot e Laurent (2001a).

${ }^{5} \mathrm{~A}$ incorporação do overnight deve-se à utilização da amostra completa para a estimação da volatilidade diária, tendo por base a relação $R_{t}=\sum R_{t, j}$ onde $R_{t}$ inclui o overnight. Adicionalmente, nos dias em que foram excluídas as primeiras cotações, os respectivos retornos são adicionados ao overnight no cálculo do primeiro retorno.
} 


\subsection{Estimação de volatilidade intradiária}

Os modelos GARCH e EGARCH utilizados nesta seção usam a série obtida a partir da amostra reduzida, composta de 3.640 retornos intradiários (intervalos de 15 minutos), no período de $3 / 4 / 2000$ a 6/10/2000. Os primeiros 1.736 retornos (62 dias, 3/4/2000 a 30/6/2000) destinam-se à estimação dos parâmetros dos modelos e dos índices de sazonalidade, enquanto que os 1.904 retornos restantes (68 dias, $3 / 7 / 2000$ a 6/10/2000) são reservados para a avaliação das estimativas. O padrão sazonal recorrente exibido pela volatilidade intradiária foi constatado para a série com a ajuda do autocorrelograma dos quadrados dos retornos. Para a avaliação da importância da sazonalidade intradiária no caso dos retornos do IBOVESPA, são utilizados inicialmente os dados brutos (desconsiderada a sazonalidade), repetindose o procedimento após a aplicação de métodos de filtragem. Para o modelo GARCH usou-se a especificação representada pelas equações:

$$
\begin{gathered}
R_{i}=a R_{i-1}+h_{i}^{\frac{1}{2}} e_{i}, \quad e_{i} \sim N I D(0,1) \\
h_{i}=\alpha_{0}+\alpha_{1} e_{i-1}^{2}+\beta h_{i-1}
\end{gathered}
$$

onde $|a|<1, \alpha_{0}>0, \alpha_{1} \geq 0$ e $\beta \geq 0$. O termo autorregressivo na média condicional busca captar a autocorrelação de primeira ordem, detectada com alta significância na série. O índice $i$ refere-se a intervalos de quinze minutos. Para o modelo EGARCH utilizou-se especificação semelhante, dada pelas equações:

$$
\begin{gathered}
R_{i}=a R_{i-1}+h_{i}^{\frac{1}{2}} e_{i}, \quad e_{i} \sim N I D(0,1) \\
\ln \left(h_{i}\right)=\alpha_{0}+\beta \ln \left(h_{i-1}\right)+\alpha_{1}\left|\frac{e_{i-1}}{h_{i-1}^{\frac{1}{2}}}\right|+\gamma \frac{e_{i-1}}{h_{i-1}^{\frac{1}{2}}}
\end{gathered}
$$

onde $\gamma$ é o parâmetro representativo da existência de assimetria na resposta da volatilidade à ocorrência de retornos positivos e negativos.

Para a filtragem da sazonalidade intradiária, Andersen e Bollerslev (1997, 1998, 1999), Taylor e Xu (1997) e Giot (2000b,a) adotam métodos determinísticos, enquanto Beltratti e Morana (1999) definem uma sazonalidade estocástica para a volatilidade intradiária. ${ }^{6}$ Optou-se por seguir a linha determinística por ser mais

\footnotetext{
${ }^{6} \mathrm{O}$ procedimento de filtragem dos retornos busca contornar a inadequação dos modelos padrão da família ARCH para acomodar os padrões fortemente cíclicos encontrados no nível intradiário. Com efeito, aqueles modelos pressupõem um decaimento geométrico na estrutura de autocorrelação dos retornos (ver Andersen e Bollerslev (1997)).
} 
simples e tendo em vista os resultados de Giot (2000), onde se constatou para este método desempenho semelhante ao obtido por procedimentos estocásticos mais complexos. Em uma primeira etapa, é necessário obter estimativas do padrão sazonal da volatilidade ao longo do dia. Segundo Taylor e Xu (1997), a soma dos quadrados dos retornos fornece uma estimativa simples de variabilidade e médias calculadas em intervalos fixos de tempo podem ser usadas para estimar o padrão sazonal. Desta forma, sendo $I_{j}$ o índice sazonal relativo ao $j$-ésimo intervalo de 15 minutos, foram estimados os 28 índices, relativos a cada um dos 28 intervalos de 15 minutos, pela média dos quadrados dos retornos verificados em cada intervalo ao longo dos 62 primeiros dias da amostra:

$$
I_{j}=\frac{1}{62} \sum_{k=1}^{62} R_{k, j}^{2} \quad j=1,2, \ldots, 28 ; \quad k=1,2, \ldots, 62
$$

Em seguida buscou-se verificar se os padrões sazonais seriam significativamente diferentes para cada dia da semana. Para isso os 62 dias foram agrupados em cinco subconjuntos, conforme o dia da semana. O mesmo procedimento foi então utilizado dando origem a 140 estimativas de índices sazonais, que refletem o comportamento da volatilidade segundo a hora do dia e o dia da semana, denotados por $I_{j, s}$ :

$$
I_{j, s}=\frac{1}{n_{s}} \sum_{k=1}^{n_{s}} R_{k, j, s}^{2}, j=1,2, \ldots, 28 ; s=1,2, \ldots, 5 ; k=1,2, \ldots, n_{s}
$$

onde $n_{s}$ é o número de vezes em que um dado dia $s$ da semana aparece durante os 62 dias considerados. Alternativamente, foram implementadas algumas modificações no procedimento acima, buscando gerar padrões de sazonalidade mais suavizados. Agora, ao invés de se considerarem apenas os dados relativos ao intervalo $j$ de 15 minutos, calculou-se $I_{j, s}$ como a média dos quadrados dos retornos verificados nos intervalos $j-1, j$ e $j+1$. No caso do primeiro e do último intervalos, foram considerados apenas os retornos dos intervalos $j ; j+1$ e $j-1 ; j$, respectivamente. Esta espécie de "janela móvel horizontal" de 45 minutos promove um alisamento inicial dos padrões de sazonalidade. A fim de se obter um conjunto de índices para intervalos de 15 minutos ainda mais suavizado, foram descartados os valores relativos aos intervalos pares e aplicou-se aos valores relativos aos intervalos ímpares um procedimento de interpolação por splines cúbicas. ${ }^{7}$ Uma vez calculadas as estimativas dos índices de sazonalidade, podemos obter a série de retornos filtrados ("dessasonalizados") por:

\footnotetext{
${ }^{7}$ Conforme proposto por Giot (2000b).
} 


$$
y_{i}=\frac{R_{i}}{\sqrt{\phi_{i}}}
$$

onde $\phi_{i}$ denota o componente determinístico de sazonalidade, que assume o valor de $I_{j}$ para o retorno $R_{i}=R_{j}$, ou seja, quando o retorno se referir ao $j$-ésimo intervalo de 15 minutos. Analogamente, para o caso em que se considerou o dia da semana, $\phi_{i}$ assume o valor de $I_{j, s}$, para o retorno $R_{i}=R_{j, s}$, ou seja, quando o retorno se referir ao intervalo $j$ do dia de semana $s$.

Uma vez construída a nova série $y_{i}$, o próximo passo consistiu na aplicação dos modelos GARCH e EGARCH. Desta forma, novos parâmetros foram estimados a partir dos retornos filtrados, que também apresentaram significativa autocorrelação de ordem 1. As especificações GARCH e EGARCH utilizadas são as mesmas dadas por (1), (2), (3) e (4). A previsão final de volatilidade condicional para ambos os modelos, reincorporando o padrão sazonal, a ser utilizada na aplicação à projeção do VaR é dada por

$$
h_{i}=\phi_{i} h_{i}^{\prime}
$$

onde $h_{i}^{\prime}$ representa a variância condicional para a série de retornos filtrados $y_{i}$.

Como parâmetro para comparação, utilizou-se o método de VaR não-paramétrico baseado no quantil empírico aplicado à série de retornos intradiários, relativos à amostra reduzida.

\subsection{Estimação de volatilidade diária com base em dados intra- diários}

Foram utilizados dois métodos na estimação da volatilidade diária a partir dos dados de alta freqüência que compõem a amostra completa. Na mesma linha adotada para os modelos da seção anterior, o conjunto amostral usado para a estimação de parâmetros é distinto do conjunto usado para a aferição dos resultados. Os parâmetros são estimados com base no período de 6/4/1998 a 29/11/2000 (402 dias e 11.256 retornos intradiários), enquanto que a aferição do desempenho abrange o período de 30/11/2000 a 19/7/2001 (400 dias e 11.200 retornos intradiários).

O primeiro método se baseia no uso de uma janela móvel para o cálculo do desvio padrão amostral. Sendo $R_{t}=\sum R_{t, j}$ e $\operatorname{var}\left(R_{t}\right)=\operatorname{var}\left(\sum R_{t, j}\right)$, então $\operatorname{var}\left(R_{t}\right)$ 
pode ser estimada diariamente por

$$
\widehat{\sigma}_{t}^{2}=\sum_{i=1}^{28} \sum_{j=1}^{28} \rho_{i, j}^{t} s_{t, i} s_{t, j}
$$

onde $\rho_{i, j}^{t}$ é o índice de correlação entre as séries de retornos verificados nos intervalos de quinze minutos de ordem $i$ e $j$ e $s_{t, i}, s_{t, j}$ são as estimativas dos respectivos desvios padrão relativos ao dia $t$. Ou seja, considerou-se que a cada intervalo $j$ de quinze minutos corresponde uma variável aleatória $R_{j}$, totalizando vinte e oito séries de retornos intradiários. Para cada variável aleatória $R_{j}$ tem-se uma amostra composta pela seqüência $\left\{R_{1, j}, R_{2, j}, \ldots, R_{802, j}\right\}, j=1,2, \ldots, 28$. Considerando-se a matriz de correlação $\left[\rho_{i, j}^{t}\right]$, de ordem $28 \times 28$ e o vetor de desvios padrão $\left[s_{t, j}\right]$, de ordem $1 \times 28$, então $\hat{\sigma}_{t}^{2}$ será dada por $s_{t}^{2}=\left[s_{t, j}\right] \mathrm{x}\left[\rho_{i, j}^{t}\right] \mathrm{x}\left[s_{t, j}\right]^{\prime}$, onde $s_{t, j^{\prime}}$ é o transposto de $\left[s_{t, j}\right]$. As estimativas dos desvios padrão $s_{t, j}$ foram calculadas pela fórmula amostral, ${ }^{8}$ utilizando uma janela móvel de 30 dias, de forma a detectar com rapidez alterações no comportamento da volatilidade. Lembrando que as 28 variáveis aleatórias $R_{j}$ formam na verdade uma única série de retornos intradiários e admitindo a hipótese de estacionariedade fraca, tem-se que o índice de correlação $\rho_{i, j}$ pode ser substituído pelo índice de autocorrelação de ordem $k=|i-j|$. Desta forma, $\left[\rho_{k}^{t}\right]$ pôde ser montada a partir do cálculo de 27 índices de autocorrelações, correspondente às 27 defasagens existentes em um dia. A janela usada para a construção de $\left[\rho_{k}^{t}\right]$ foi de 402 dias. Assim, as estimativas $s_{t}^{2}$ são computadas a partir do $403^{\circ}$ dia da amostra, com base nos dados referentes aos 402 dias anteriores. No passo seguinte, a previsão de volatilidade diária $s_{t}$, dada pela raiz quadrada de $s_{t}^{2}$, é utilizada no cálculo do VaR. A matriz $\left[\rho_{k}^{t}\right]$ é recalculada diariamente, totalizando 400 matrizes diferentes, onde a janela móvel de 402 dias (11.256 retornos) é mantida. Dada a estacionariedade dos retornos intradiários, é razoável supor que o recálculo de $\left[\rho_{k}^{t}\right]$ em intervalos maiores não afete significativamente o resultado. Desta forma, repetiu-se o procedimento ampliando o intervalo de recálculo para 20 dias. $^{9}$ Adicionalmente, substituiu-se $\left[\rho_{k}^{t}\right]$ pela matriz identidade. Com isto buscou-se avaliar o impacto da hipótese de estacionariedade para a série de retornos intradiários e do decorrente uso de $\left[\rho_{k}^{t}\right]$.

O segundo método utilizado se baseia no uso de alisamento exponencial, consistindo em uma adaptação do procedimento descrito no documento Riskme-

${ }^{8}$ Dada por $S_{t, j}=\left[\frac{1}{29} \sum_{t=1}^{30}\left(R_{t, j}-\mu_{R_{j}}\right)^{2}\right]^{\frac{1}{2}}$.

${ }^{9}$ Contudo, $\left[s_{t, j}\right]$ permanece sendo recalculado diariamente com janela móvel de 30 dias. 
trics Group (1996), em sua versão para média de retornos igual a zero, com um parâmetro de decaimento $\lambda=0,94$ para todas as séries $R_{t, j}{ }^{10}$

Assim, para cada uma das 28 séries de retornos de 15 minutos, a variância condicional no dia t relativa ao $j$-ésimo intervalo de quinze minutos é dada por

$$
h_{t, j}=\lambda h_{t-1, j}+(1-\lambda) R_{t-1, j}^{2}
$$

onde $\lambda$ é o parâmetro de decaimento e $R_{t-1, j}^{2}$ é o quadrado do respectivo retorno observado no dia anterior. Uma vez obtidas as estimativas de variância condicional para cada dia $t$ e intervalo $j$ de quinze minutos, calculou-se a variância condicional diária, pela fórmula $s_{t}^{2}=\left[s_{t, j}\right] \times\left[\rho_{k}^{t}\right] \mathrm{x}\left[s_{t, j}\right]^{\prime}$, onde $\left[\rho_{k}^{t}\right]$ é a mesma matriz de autocorrelação utilizada no método anterior e $\left[s_{t, j}\right]$ é formado pelos desvios padrão condicionais calculados como a raiz quadrada das variâncias condicionais $h_{t, j}$. A previsão de volatilidade diária $s_{t}$ é, então, utilizada para o cálculo do VaR diário. Analogamente ao caso anterior, avaliou-se o cálculo da matriz de autocorrelação em intervalos de 20 dias, bem como se substituiu $\left[\rho_{k}^{t}\right]$ pela matriz identidade.

Para uma melhor avaliação destes métodos, foi também calculada a estimativa de volatilidade diária, para o mesmo período, utilizando-se a série diária de retornos, obtida com base apenas nos preços de fechamento. Os métodos escolhidos foram: desvio padrão com janela móvel de 30 dias, alisamento exponencial, GARCH e EGARCH. O desvio padrão é dado pela fórmula amostral e os demais modelos seguem as mesmas especificações dadas pelas equações (1) a (4) e (10).

\subsection{Cálculo e aferição das projeções de VaR}

Foram computados os valores em risco relativos aos percentis de 1\%, 2,5\% e $5 \%$ (cauda esquerda) e $95 \%, 97,5 \%$ e $99 \%$ (cauda direita), para todas as situações consideradas. O desempenho das projeções de VaR é aferido com base no teste de Kupiec (1995) para a proporção de vezes em que o quantil previsto é ultrapassado. Admitindo que os distúrbios aleatórios da série são normalmente distribuídos, usou-se para os métodos paramétricos a fórmula (11):

$$
\operatorname{VaR}_{t}(\alpha \%)=\exp \left(s_{t} z_{\alpha \%}\right)-1
$$

onde $z_{\alpha}$ corresponde ao quantil da normal padronizada cuja área sob a curva de

\footnotetext{
${ }^{10}$ Apesar de consistir no padrão Riskmetrics para o mercado americano, o parâmetro de decaimento $\lambda=0,94$ é também comumente usado no mercado brasileiro e já foi testado em alguns artigos (ver Lemgruber e Ohanian (2001).
} 
densidade à sua direita é $\alpha \%$ e $s_{t}$ é a estimativa de volatilidade. ${ }^{11}$ Assim, o valor em risco é obtido na forma de retorno (e não em valor monetário), podendo ser diretamente comparado à série de retornos efetivos, calculados por $P_{i} / P_{i-1}-1$, para efeito de aferição. No método não-paramétrico baseado no quantil empírico, empregado na seção 2.1 , o $\operatorname{VaR}(\alpha \%)$ é dado diretamente pelo quantil $\mathrm{Q}(\alpha \%)$ da série composta pelos últimos m retornos efetivos de 15 minutos. Foram adotadas janelas móveis de $\mathrm{m}=840$ observações e $\mathrm{m}=1.680$ observações (equivalendo a 30 e 60 dias, respectivamente).

São considerados como falhas os eventos em que o retorno verificado é inferior (maior em módulo) que o previsto pelo VaR na cauda esquerda, ou maior que o previsto na cauda direita. Espera-se que a proporção verificada de falhas em relação ao total de observações esteja próxima de $\alpha \%$, que corresponde ao limite arbitrado (proporção teórica) para avaliação do desempenho dos modelos.

Aplicou-se às séries de projeções de VaR o teste de Kupiec para a proporção de falhas. Foram construídos intervalos com $95 \%$ de confiança para a não rejeição da hipótese nula para a proporção empírica de vezes em que o VaR foi ultrapassado. ${ }^{12}$ Quando a proporção verificada em cada caso esteve contida no respectivo intervalo, H0 não foi rejeitada. Adicionalmente calculamos o p-valor para as proporções encontradas.

\section{Resultados}

Esta seção apresenta os resultados organizados em dois blocos. O primeiro mostra o comportamento da sazonalidade intradiária para o IBOVESPA e o efeito da filtragem dos padrões observados no desempenho do VaR intradiário, tendo por base a amostra reduzida. O bloco seguinte mostra os resultados do uso de dados intradiários para cálculo do VaR diário, com base na amostra completa.

\subsection{Aferição do VaR intradiário}

A tabela 1 permite comparar algumas estatísticas básicas para as séries de retornos diários e intradiários. A exemplo do que ocorre com a série diária, a série

\footnotetext{
${ }^{11} \mathrm{Ou}$ seja, $z_{\alpha} \%$ é igual a $2,32 \quad 1,96$ e 1,64, aproximadamente, para $\alpha \%$ igual a $1 \%, 2,5 \%$ e $5 \%$, respectivamente. O uso da função exponencial na fórmula do VaR deve-se ao uso de retornos logarítmicos no cálculo das estimativas de volatilidade. Com efeito, $s_{t} . z_{\alpha}$ representa um quantil da distribuição de retornos logarítmicos.

${ }^{12}$ Testa-se a hipótese nula de que a probabilidade empírica de se observar falhas é igual ao nível pré-especificado para o VaR.
} 
de retornos de alta freqüência relativa à amostra completa apresenta alto índice de curtose. Cabe ressaltar a forte redução deste índice, de 28,16 para 7,48, quando consideramos a amostra reduzida, onde o overnight é excluído. Esta redução é ainda mais expressiva após o procedimento de filtragem dos retornos, com o índice de curtose chegando a 2,16. A existência de aglomerados de volatilidade pode ser inferida com base no autocorrelograma dos quadrados dos retornos da série intradiária (gráfico 1). Nota-se, também, um forte e distintivo padrão sazonal para o processo de volatilidade em formato de "J invertido", com valores expressivos no início do dia, decrescendo até meados do período diário e voltando a crescer em seguida até o final do dia, denotando a existência de sazonalidade intradiária no processo de volatilidade. Tal padrão pode refletir, entre outros fatores, as informações públicas acumuladas entre o fechamento do pregão em um dia e a abertura no dia seguinte, bem como a menor atividade em torno da hora do almoço. O gráfico 2 retrata os índices de sazonalidade calculados para cada intervalo de 15 minutos e confirma, para a amostra reduzida, a existência do padrão intradiário. Já o gráfico 3 indica que pode haver uma influência significativa do dia da semana no comportamento da volatilidade, haja vista os diferentes padrões observados. Porém, é possível identificar um padrão geral caracterizado por "picos" no início e "vales" no meio do período diário, para todos os dias. Pode-se ainda observar no gráfico 4 o resultado da técnica de alisamento usada nas estimativas dos padrões sazonais, cujas formas mais suavizadas contrastam com as anteriores. É interessante, por fim, avaliar o efeito do procedimento de filtragem aplicado à amostra reduzida. $\mathrm{O}$ gráfico 5 mostra que o padrão sazonal foi removido em boa parte, a exemplo do que registraram Andersen e Bollerslev (1997, 1998b), Beltratti e Morana (1999), Andersen (2000) e Giot (2000b). Uma comparação com o autocorrelograma dos quadrados dos retornos diários, exibido no gráfico 6 , permite constatar para a série de alta freqüência um decaimento na estrutura de autocorrelação mais próximo dos padrões modelados adequadamente por modelos da família ARCH.

Os números relatados nas tabelas 2 e 3 mostram que o uso dos modelos GARCH e EGARCH com dados de alta freqüência cujo fator sazonal intradiário não foi filtrado geram estimativas de volatilidade e projeções de VaR sem utilidade. Somente após a devida incorporação do fenômeno, os modelos tornaram-se efetivos. O modelo GARCH apresenta projeções de VaR demasiadamente conservadoras quando aplicado diretamente aos dados não filtrados, como mostra a tabela 2. Com efeito, a hipótese nula só é mantida para o percentil de $1 \%$ em ambas as caudas, sendo rejeitada para os demais níveis pré-especificados para o VaR. Há uma sensível melhora quando se leva em conta a sazonalidade. Numa primeira etapa, a filtragem se dá apenas em termos dos intervalos de 15 minutos ao longo do pregão, despre- 
zando o eventual efeito do dia de semana. Neste caso a hipótese nula é rejeitada apenas para o percentil de $5 \%$, em ambas as caudas. Ao se considerar também o efeito do dia da semana, mas sem a aplicação de qualquer procedimento de alisamento, não há uma melhora consistente, em temos gerais, em relação à situação anterior. A hipótese nula passa a ser mantida para $5 \%$ na cauda direita, mas é rejeitada para $1 \%$.

Tabela 1

Estatísticas Descritivas Básicas da Série de Retornos Logarítmicos do IBOVESPA Períodos de 7/4/1998 a 19/7/2001 (amostra completa - retornos diários e intradiários) e 3/4/2000 a 6/10/2000 (amostra reduzida - retornos intradiários - não filtrados e filtrados)

\begin{tabular}{|c|c|c|c|c|c|c|c|}
\hline & Média & Mediana & Máximo & Mínimo & Desvio Padrão & Curtose & Assimetria \\
\hline $\begin{array}{l}\text { Diários: Amostra } \\
\text { Completa }^{(a)}\end{array}$ & 0,000175 & 0,000160 & 0,288248 & $-0,172377$ & 0,02921 & 15,919 & 1,005 \\
\hline $\begin{array}{l}\text { Intradiários: } \\
\text { Amostra } \\
\text { Completa }^{(b)}\end{array}$ & 0,000006 & 0,0000166 & 0,075707 & $-0,076488$ & 0,004349 & 28,164 & $-0,034$ \\
\hline $\begin{array}{l}\text { Intradiários: } \\
\text { Amostra } \\
\text { Reduzida }^{(c)}\end{array}$ & $-0,000039$ & $-0,000043$ & 0,022237 & $-0,028513$ & 0,00343 & 7,481 & $-0,357$ \\
\hline $\begin{array}{l}\text { Intradiários: } \\
\text { Amostra } \\
\text { Reduzida } \\
\text { Filtrada }^{(d)}\end{array}$ & $-0,014636$ & $-0,013410$ & 4,116715 & $-4,920502$ & 0,882680 & 2,155 & $-0,076932$ \\
\hline
\end{tabular}

(a) 802 dados;

(b) 22.456 dados;

(c) e (d) 3.640 dados.

Gráfico 1

Função de autocorrelação da série de quadrados nos logaritmicos de 15 minutos do Ibovespa no período de 7/4/1998 a 19/7/2001

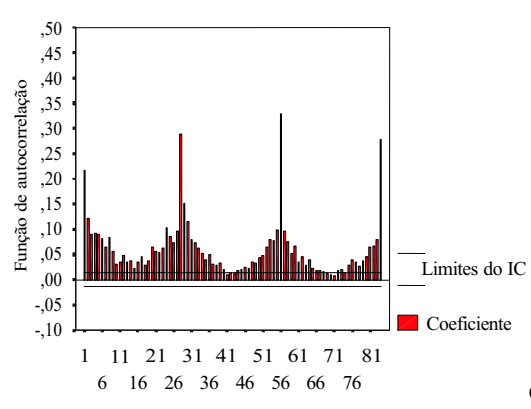

Defasagem em intervalos de 15 minutos
Gráfico 2

Padrão bruto de sazonalidade dos retornos logarítmicos intradiários do Ibovespa por intervalos de 15 minutos ndices estimados com base no período de $3 / 4 / 2000$ a $30 / 6 / 2000$

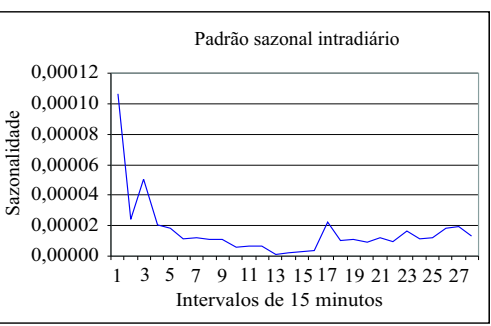

Obs.: os índices consideram apenas os intervalos de 15 minutos ao longo do pregão diário, não levando em conta o dia da semana em que ocorrem. O padrão sazonal resultante não passou por processo de alisamento. 
Gráfico 3
Padrões brutos de sazonalidade da volatilidade dos retornos logarítmicos de 15 minutos do Ibovespa por dia da semana - Índices estimados com base no período de 3/4/2000 a 30/6/2000

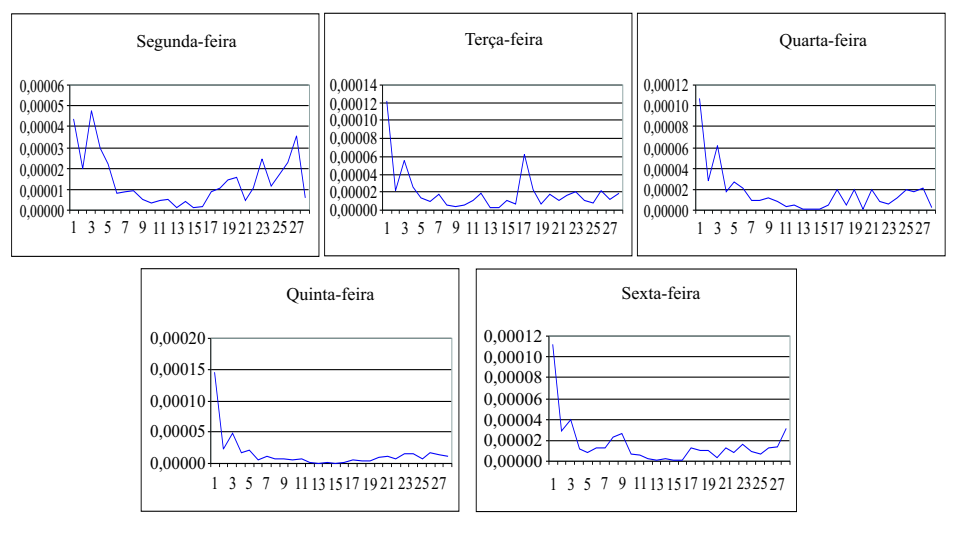

Obs:: Os padrões sazonais de volatilidade (eixo das ordenadas) são estimados com base na média dos quadrados dos retornos verificados em intervalos de 15 minutos (eixo das abscissas).

Gráfico 4

Padrões brutos de sazonalidade da volatilidade dos retornos logarítmicos de 15 minutos do Ibovespa por dia da semana, após interpolação por splines cúbicas indices estimados com base no período de 3/4/2000 a 30/6/2000

\begin{tabular}{|c|c|c|c|c|c|}
\hline \multirow{2}{*}{\multicolumn{2}{|c|}{ Segunda-feira }} & \multicolumn{2}{|r|}{ Terça-feira } & \multicolumn{2}{|r|}{ Ouarta-feira } \\
\hline & & 0,00008 & & $\mid 0,00008$ & \\
\hline 0,00003 & & 0,00006 & & || $0,00006$ & \\
\hline $\begin{array}{l}0,00002 \\
0,00002\end{array}$ & 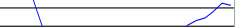 & 0,00004 & & $\mid 0,00004$ & \\
\hline 0,00001 & $\sim$ & 0,00002 & $\triangle$ & $\mid 0,00002$ & \\
\hline 0,00000 & 4 & 0,00000 & $>$ & 0,00000 & \\
\hline & 13579111315171921232527 & & 135579111315171921232527 & & 13579111315171921232527 \\
\hline
\end{tabular}

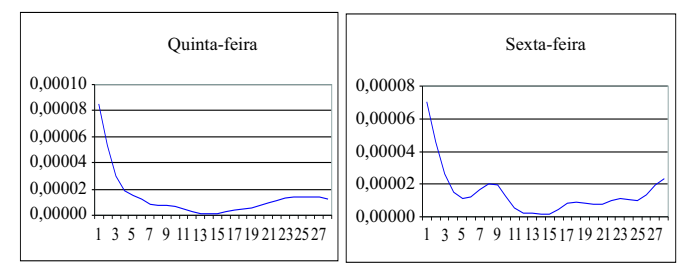

Obs:: Os padrões sazonais de volatilidade (eixo das ordenadas) são estimados com base na média dos quadrados dos retornos verificados em intervalos de 15 minutos (eixo das abscissas). 

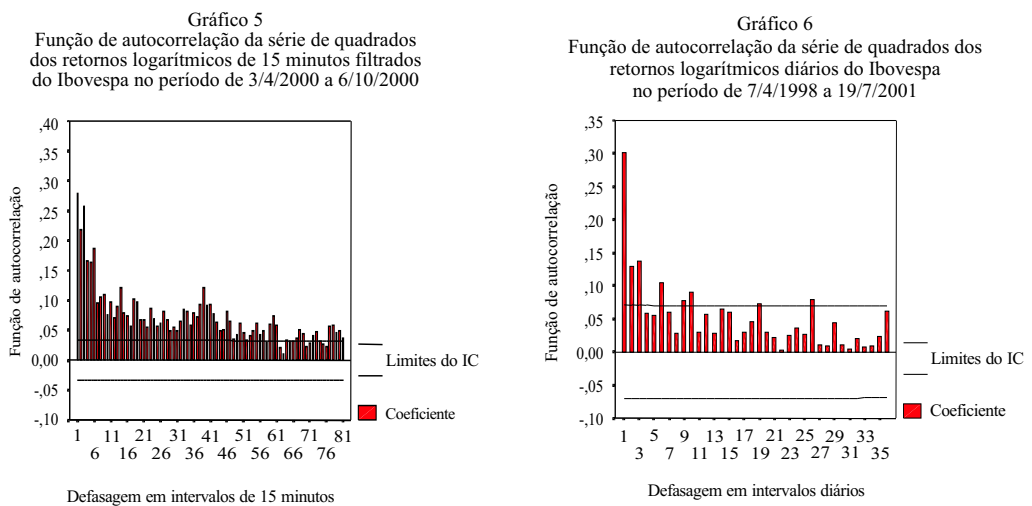

Quando se aplica o procedimento de alisamento à série de índices sazonais, os resultados apresentam uma melhora em relação ao desempenho das projeções obtidas anteriormente, marcadamente no tocante à posição vendida. Agora, a rejeição ocorre apenas para $5 \%$ na posição comprada como pode ser constatado na tabela 2 .

Tabela 2

Avaliação das Estimativas de VaR Intradiário (15 minutos) a partir de Estimativas de Volatilidade Geradas pela Aplicação do Modelo GARCH $(1,1)$ à Série dos Retornos Logarítmicos do IBOVESPA - Período de 3/4/2000 a 6/10/2000 (amostra reduzida - dados intradiários)

\begin{tabular}{|c|c|c|c|c|c|c|c|c|}
\hline \multirow{2}{*}{\multicolumn{2}{|c|}{ Probabilidade do VaR }} & & \multicolumn{3}{|c|}{ Cauda Esquerda } & \multicolumn{3}{|c|}{ Cauda Direita } \\
\hline & & & $1 \%$ & $2,5 \%$ & $5 \%$ & $1 \%$ & $2,5 \%$ & $5 \%$ \\
\hline \multirow{3}{*}{\multicolumn{2}{|c|}{$\begin{array}{c}\text { Dados Brutos - } \\
\text { Sazonalidade } \\
\text { Intradiária não Filtrada }\end{array}$}} & Proporção & & & & & & \\
\hline & & Empírica & $0,79 \%$ & $1,68 \%$ & $3,10 \%$ & $1,10 \%$ & $1,63 \%$ & $3,26 \%$ \\
\hline & & p-valor & 0,33385 & 0,01500 & 0,00005 & 0,65698 & 0,00933 & 0,00020 \\
\hline \multirow{3}{*}{\multicolumn{2}{|c|}{$\begin{array}{l}\text { Sazonalidade Filtrada } \\
\text { por Período de } 15 \text { min. } \\
\text { do dia }\end{array}$}} & Proporção & & & & & & \\
\hline & & Empírica & $1,05 \%$ & $2,15 \%$ & $3,73 \%$ & $0,89 \%$ & $2,31 \%$ & $3,36 \%$ \\
\hline & & p-valor & 0,82641 & 0,3212 & 0,00782 & 0,63224 & 0,59255 & 0,00050 \\
\hline \multirow{5}{*}{$\begin{array}{l}\text { Sazonalidade } \\
\text { Filtrada por } \\
\text { Período de } 15 \\
\text { min. do Dia e } \\
\text { por Dia da } \\
\text { Semana }\end{array}$} & Sem & Proporção & & & & & & \\
\hline & \multirow[b]{2}{*}{ Alisamento } & Empírica & $1,31 \%$ & $2,57 \%$ & $3,89 \%$ & $1,89 \%$ & $3,20 \%$ & $4,46 \%$ \\
\hline & & p-valor & 0,19027 & 0,83794 & 0,02056 & 0,00051 & 0,05926 & 0,27502 \\
\hline & \multirow[b]{2}{*}{ Alisamento } & $\begin{array}{l}\text { Proporção } \\
\text { Empírica }\end{array}$ & $1,16 \%$ & $2,10 \%$ & $3,47 \%$ & $1,10 \%$ & $2,47 \%$ & $4,05 \%$ \\
\hline & & p-valor & 0,50534 & 0,25153 & 0,00119 & 0,65698 & 0,92967 & 0,0500 \\
\hline
\end{tabular}

Os resultados do modelo EGARCH, dispostos na tabela 3, apresentam compor- 
tamento análogo ao verificado para o modelo GARCH. A melhora do desempenho após a filtragem do padrão sazonal é evidente. No caso em que a sazonalidade é filtrada desconsiderando-se o dia da semana os resultados são superiores, com a rejeição da hipótese nula, $H 0$, apenas para $5 \%$ na cauda esquerda. O procedimento adicional de filtragem por dia da semana gerou uma deterioração das projeções de VaR, levando à rejeição de $\mathrm{H} 0$ para $1 \%$ em ambas as caudas. O alisamento dos padrões sazonais promoveu uma recuperação parcial das projeções com filtragem por dia da semana, tornando a ocorrer rejeição de $\mathrm{H} 0$ apenas para $5 \%$ da cauda esquerda.

Tabela 3

Avaliação das Estimativas de VaR Intradiário (15 minutos) a partir de Estimativas de Volatilidade Geradas pela Aplicação do Modelo EGARCH $(1,1)$ à Série dos Retornos Logarítmicos do IBOVESPA - Período de 3/4/2000 a 6/10/2000 (amostra reduzida dados intradiários)

\begin{tabular}{|c|c|c|c|c|c|c|c|c|}
\hline \multirow{2}{*}{\multicolumn{2}{|c|}{ Probabilidade do VaR }} & & \multicolumn{3}{|c|}{ Cauda Esquerda } & \multicolumn{3}{|c|}{ Cauda Direita } \\
\hline & & & $1 \%$ & $2,5 \%$ & $5 \%$ & $1 \%$ & $2,5 \%$ & $5 \%$ \\
\hline \multirow{3}{*}{\multicolumn{2}{|c|}{$\begin{array}{c}\text { Dados Brutos - } \\
\text { Sazonalidade } \\
\text { Intradiária não Filtrada }\end{array}$}} & Proporção & & & & & & \\
\hline & & Empírica & $0,74 \%$ & $1,68 \%$ & $2,84 \%$ & $1,10 \%$ & $1,79 \%$ & $3,26 \%$ \\
\hline & & p-valor & 0,22317 & 0,01500 & 0,00000 & 0,65698 & 0,03554 & 0,00020 \\
\hline \multirow{3}{*}{\multicolumn{2}{|c|}{$\begin{array}{l}\text { Sazonalidade Filtrada } \\
\text { por Período de } 15 \text { min. } \\
\text { do dia }\end{array}$}} & Proporção & & & & & & \\
\hline & & Empírica & $1,21 \%$ & $2,31 \%$ & $3,83 \%$ & $1,00 \%$ & $2,63 \%$ & $4,15 \%$ \\
\hline & & p-valor & 0,37710 & 0,59255 & 0,01510 & 0,99265 & 0,72674 & 0,07962 \\
\hline \multirow{4}{*}{$\begin{array}{l}\text { Sazonalidade } \\
\text { Filtrada por } \\
\text { Período de } 15 \\
\text { min. do Dia e } \\
\text { por Dia da } \\
\text { Semana }\end{array}$} & \multirow[b]{2}{*}{ Alisamento } & \begin{tabular}{|l} 
Proporção \\
Empírica
\end{tabular} & $1,58 \%$ & $2,78 \%$ & $4,25 \%$ & $2,15 \%$ & $3,20 \%$ & $4,62 \%$ \\
\hline & & $\mathrm{p}$-valor & 0,01987 & 0,43615 & 0,12583 & 0,00001 & 0,05926 & 0,44341 \\
\hline & \multirow[b]{2}{*}{ Alisamento } & $\begin{array}{l}\text { Proporção } \\
\text { Empírica }\end{array}$ & $1,26 \%$ & $2,36 \%$ & $3,78 \%$ & $1,31 \%$ & $2,78 \%$ & $4,41 \%$ \\
\hline & & p-valor & 0,27220 & 0,70015 & 0,01094 & 0,19027 & 0,43615 & 0,22985 \\
\hline
\end{tabular}

A tabela 4 exibe os resultados do método não paramétrico do quantil empírico, adotado para efeito de comparação. O desempenho do VaR com base na janela de 840 observações (30 dias) apresentou resultados bastante bons, não ocorrendo a rejeição de H0 para qualquer das probabilidades avaliadas. ${ }^{13}$ Já o desempenho obtido a partir da janela de 1680 observações (60 dias) é fraco, ocorrendo a rejeição de $\mathrm{H} 0$ para $5 \%$ na cauda esquerda e $2,5 \%$ e $5 \%$ na cauda direita.

\footnotetext{
${ }^{13}$ Giot (2000a) também constatou o bom desempenho deste método para retornos de alta freqüência, tendo em vista levar explicitamente em conta a existência de caudas pesadas para a série.
} 
Tabela 4

Avaliação das Estimativas de VaR Intradiário (15 minutos) a partir de Estimativas de Volatilidade Geradas pela Aplicação do Método não Paramétrico Baseado no Quantil Empírico à Série dos Retornos do IBOVESPA - Período de 3/4/2000 a 6/10/2000 (amostra reduzida - dados intradiários)

\begin{tabular}{|c|c|c|c|c|c|c|c|}
\hline & & \multicolumn{3}{|c|}{ Cauda Esquerda } & \multicolumn{3}{|c|}{ Cauda Direita } \\
\hline \multicolumn{2}{|c|}{ Probabilidade do VaR } & $1 \%$ & $2,5 \%$ & $5 \%$ & $1 \%$ & $2,5 \%$ & $5 \%$ \\
\hline \multirow{2}{*}{$\begin{array}{c}\text { Janela de } 840 \\
\text { observações (30 dias) }\end{array}$} & \begin{tabular}{|c|} 
Proporção \\
Empírica
\end{tabular} & $1,10 \%$ & $2,89 \%$ & $5,67 \%$ & $0,95 \%$ & $2,42 \%$ & $5,09 \%$ \\
\hline & p-valor & 0,656978 & 0,288934 & 0,187206 & 0,808972 & 0,813306 & 0,850317 \\
\hline \multirow{2}{*}{$\begin{array}{c}\text { Janela de } 1680 \\
\text { observações (60 dias) }\end{array}$} & $\begin{array}{c}\text { Proporção } \\
\text { Empírica }\end{array}$ & $0,68 \%$ & $2,10 \%$ & $4,04 \%$ & $0,68 \%$ & $1,68 \%$ & $3,99 \%$ \\
\hline & p-valor & 0,140003 & 0,251526 & 0,048067 & 0,140003 & 0,014999 & 0,036672 \\
\hline
\end{tabular}

\subsection{Aferição do VaR diário com base em dados intradiários}

Os resultados alcançados nesta seção indicam que é possível obter projeções adequadas para a volatilidade e para o VaR diário a partir do uso de dados intradiários. A avaliação do método baseado no desvio padrão com janela móvel aplicado aos retornos de alta frequência para a obtenção de estimativas de volatilidade e projeção de VaR diários pode ser acompanhada na tabela 5. A hipótese nula é aceita para todos os níveis de segurança pré-especificados para o VaR, tanto para a posição comprada como para a posição vendida. Contudo, o desempenho relativo à cauda esquerda é significativamente superior, o que pode ser constatado através dos p-valores. Nota-se ainda que o desempenho é relativamente mais fraco para o percentil mais extremo, de $1 \%$, em ambos os casos. Conforme esperado, o recálculo da matriz de autocorrelação de 20 em 20 dias, ao invés de diariamente, não influiu nos resultados. A substituição da matriz de autocorrelação pela matriz identidade ocasionou forte deterioração dos resultados, marcadamente para a cauda esquerda onde todas as proporções empíricas ficam fora do intervalo de aceitação de H0. 
Tabela 5

Avaliação das Estimativas de VaR Diário a partir de Estimativas de Volatilidade Geradas pela Aplicação do Método Baseado no Desvio Padrão com Janela Móvel de 30 Dias à Série dos Retornos Logarítmicos Intradiários (15 minutos) do IBOVESPA Período de 7/4/1998 a 19/7/2001 (amostra completa - dados intradiários)

\begin{tabular}{|c|c|c|c|c|c|c|c|}
\hline & & \multicolumn{3}{|c|}{ Cauda Esquerda } & \multicolumn{3}{|c|}{ Cauda Direita } \\
\hline \multicolumn{2}{|l|}{ Probabilidade do VaR } & $1 \%$ & $2,5 \%$ & $5 \%$ & $1 \%$ & $2,5 \%$ & $5 \%$ \\
\hline \multirow{3}{*}{$\begin{array}{c}\text { Com Recálculo Diário } \\
\text { da Matriz de } \\
\text { Autocorrelação }\end{array}$} & Proporção & & & & & & \\
\hline & Empírica & $1,50 \%$ & $2,50 \%$ & $5,25 \%$ & $0,25 \%$ & $1,75 \%$ & $4,00 \%$ \\
\hline & p-valor & 0,34938 & 1,00000 & 0,81993 & 0,07142 & 0,31026 & 0,34242 \\
\hline \multirow{3}{*}{$\begin{array}{c}\text { Com Recálculo da } \\
\text { Matriz de Autocorrelação } \\
\text { a cada } 20 \text { dias }\end{array}$} & Proporção & & & & & & \\
\hline & Empírica & $1,50 \%$ & $2,50 \%$ & $5,25 \%$ & $0,25 \%$ & $1,75 \%$ & $4,00 \%$ \\
\hline & p-valor & 0,34938 & 1,00000 & 0,81993 & 0,07142 & 0,31026 & 0,34242 \\
\hline \multirow{3}{*}{$\begin{array}{l}\text { Com a Matriz de } \\
\text { Autocorrelação } \\
\text { Substituída pela } \\
\text { Matriz Identidade }\end{array}$} & Proporção & & & & & & \\
\hline & Empírica & $2,50 \%$ & $4,75 \%$ & $8,50 \%$ & $1,25 \%$ & $3,75 \%$ & $6,00 \%$ \\
\hline & p-valor & 0,01130 & 0,01020 & 0,00335 & 0,62860 & 0,13550 & 0,37299 \\
\hline
\end{tabular}

Os resultados do método baseado em alisamento exponencial são semelhantes aos resultados do método anterior, como mostra a tabela 6. A hipótese nula também é aceita em todas as situações, com desempenho claramente superior para as projeções de VaR para a posição comprada. Analogamente ao caso anterior, o uso da matriz identidade leva a uma piora considerável do desempenho geral do modelo. A melhora relativa das proporções referentes à posição vendida não compensa a forte deterioração constatada para a posição comprada.

Tabela 6

Avaliação das Estimativas de VaR Diário a partir de Estimativas de Volatilidade Geradas pela Aplicação do Método Baseado em Alisamento Exponencial à Série dos Retornos Logarítmicos Intradiários (15 minutos) do IBOVESPA Período de 7/4/1998 a 19/7/2001 (amostra completa - dados intradiários)

\begin{tabular}{|c|c|c|c|c|c|c|c|}
\hline & & \multicolumn{3}{|c|}{ Cauda Esquerda } & \multicolumn{3}{|c|}{ Cauda Direita } \\
\hline \multicolumn{2}{|l|}{ Probabilidade do VaR } & $1 \%$ & $2,5 \%$ & $5 \%$ & $1 \%$ & $2,5 \%$ & $5 \%$ \\
\hline \multirow{2}{*}{$\begin{array}{c}\text { Com Recálculo Diário } \\
\text { da Matriz de } \\
\text { Autocorrelação }\end{array}$} & Proporção & $1.50 \%$ & $250 \%$ & $475 \%$ & $0.50 \%$ & $125 \%$ & $3.75 \%$ \\
\hline & p-valor & 0,34938 & 1,00000 & 0,81711 & 0,26595 & 0,07675 & 0,23094 \\
\hline \multirow{3}{*}{$\begin{array}{l}\text { Com Recálculo da } \\
\text { Matriz de Autocorrelação } \\
\text { a cada } 20 \text { dias }\end{array}$} & Proporção & & & & & & \\
\hline & Empírica & $1,50 \%$ & $2,50 \%$ & $4,75 \%$ & $0,50 \%$ & $1,25 \%$ & $3,75 \%$ \\
\hline & p-valor & 0,34938 & 1,00000 & 0,81711 & 0,26595 & 0,07675 & 0,23094 \\
\hline \multirow{2}{*}{$\begin{array}{l}\text { Com a Matriz de } \\
\text { Autocorrelação } \\
\text { Substituída pela } \\
\text { Matriz Identidade }\end{array}$} & $\begin{array}{l}\text { Proporção } \\
\text { Empírica }\end{array}$ & $2,50 \%$ & $4,50 \%$ & $7,75 \%$ & $0,75 \%$ & $3,25 \%$ & $5,50 \%$ \\
\hline & p-valor & 0,01130 & 0,02102 & 0,01909 & 0,59905 & 0,35808 & 0,65136 \\
\hline
\end{tabular}


A tabela 7 reúne os resultados de alguns modelos tradicionais de estimação de volatilidade e projeção de VaR aplicados a retornos diários, escolhidos para uma comparação com os métodos avaliados nas tabelas 5 e 6 .

O método de desvio padrão com janela móvel de dados e o de alisamento exponencial com $\lambda=0,94$ obtiveram um bom desempenho para a cauda direita, sendo este último o detentor dos melhores resultados em termos gerais. Já os dois modelos da família GARCH mostraram bom desempenho para a cauda esquerda, com destaque para o GARCH $(1,1)$, contrastando com o fraco desempenho da cauda direita. Nenhum dos dois passou no teste de Kupiec para o nível de segurança de $5 \%$ para a posição vendida. Em termos gerais, o desempenho mais fraco deveu-se ao modelo EGARCH $(1,1)$, apesar da alta significância encontrada para o parâmetro indicativo de assimetria e alavancagem.

Em relação aos métodos baseados em dados intradiários, é possível verificar naqueles um desempenho semelhante ao do EWMA e levemente superior ao método de janela móvel de dados (estes com base em dados diários). Já os modelos GARCH e EGARCH se mostraram claramente inferiores.

Tabela 7

Avaliação das Estimativas de VaR Diário a partir de Estimativas de Volatilidade Geradas pela Aplicação de Métodos Tradicionais à Série dos Retornos Logarítmicos Diários do

IBOVESPA - Período de 7/4/1998 a 19/7/2001 (amostra completa - dados diários)

\begin{tabular}{c|cccc|ccc}
\hline & \multicolumn{4}{c|}{ Cauda Esquerda } & \multicolumn{4}{c}{ Cauda Direita } \\
\hline Probabilidade do VaR & \multicolumn{1}{c}{$1 \%$} & $2,5 \%$ & $5 \%$ & $1 \%$ & $2,5 \%$ & $5 \%$ \\
\hline $\begin{array}{c}\text { Desvio Padrão com } \\
\text { Janela Móvel } \\
\text { de 30 dias }\end{array}$ & $\begin{array}{c}\text { Proporção } \\
\text { Empírica }\end{array}$ & $2,00 \%$ & $3,75 \%$ & $6,75 \%$ & $0,75 \%$ & $3,00 \%$ & $5,25 \%$ \\
\cline { 2 - 8 } & p-valor & 0,07682 & 0,13550 & 0,12646 & 0,59905 & 0,53441 & 0,81993 \\
\hline $\begin{array}{c}\text { Alisamento } \\
\text { Exponencial com } \\
\lambda=0,94\end{array}$ & $\begin{array}{c}\text { Proporção } \\
\text { Empírica }\end{array}$ & $1,75 \%$ & $3,5 \%$ & $6,00 \%$ & $0,75 \%$ & $2,25 \%$ & $4,75 \%$ \\
\cline { 2 - 8 } & p-valor & 0,17293 & 0,22655 & 0,37299 & 0,59905 & 0,74460 & 0,81711 \\
\hline $\begin{array}{c}\text { GARCH } \\
(1,1)\end{array}$ & $\begin{array}{c}\text { Proporção } \\
\text { Empírica }\end{array}$ & $0,75 \%$ & $2,50 \%$ & $4,00 \%$ & $0,25 \%$ & $1,25 \%$ & $2,25 \%$ \\
\cline { 2 - 8 } & p-valor & 0,59905 & 1,00000 & 0,34242 & 0,07142 & 0,07675 & 0,00483 \\
\hline \multirow{2}{*}{$\begin{array}{c}\text { EGARCH } \\
(1,1)\end{array}$} & $\begin{array}{c}\text { Proporção } \\
\text { Empírica }\end{array}$ & $0,75 \%$ & $1,75 \%$ & $3,50 \%$ & $0,25 \%$ & $1,25 \%$ & $2,25 \%$ \\
\cline { 2 - 7 } & p-valor & 0,59905 & 0,31026 & 0,14659 & 0,07142 & 0,07675 & 0,00483 \\
\hline
\end{tabular}

Os p-valores iguais ou maiores que 0,05 indicam que a hipótese nula, de que a verdadeira proporção de falhas do modelo é igual ao nível pré-especificado para o VaR, não pode ser rejeitada ao nível de significância de $5 \%$ do teste.

\section{Conclusão}

Este trabalho busca extrair algumas conclusões sobre a estimação de volatilidades diária e intradiária, e seu uso em métodos de cálculo do valor em risco, a partir da utilização de uma série de dados de alta freqüência do IBOVESPA. 
O padrão em forma de "J invertido" exibido pela função de autocorrelação dos quadrados dos retornos intradiários é fortemente distintivo em relação a séries de retornos diários. O uso de retornos de alta freqüência nos modelos de volatilidade GARCH e EGARCH, sem o tratamento prévio do fator sazonal, gera fortes distorções na previsão da volatilidade intradiária. A filtragem da sazonalidade promove uma forte melhora nos resultados, comprovando ser indispensável sua aplicação ao uso daqueles modelos. Ambos os modelos passaram no teste de $\mathrm{Ku}-$ piec com $5 \%$ de significância, para os níveis de $1 \%$ e $2,5 \%$ na cauda esquerda e $1 \%$, $2,5 \%$ e $5 \%$ na cauda direita, com rejeição da hipótese nula apenas para o nível de $5 \%$ da cauda esquerda. O procedimento de filtragem buscando levar em conta o dia da semana em que os retornos ocorrem não contribui significativamente para melhorar os resultados, em relação à simples filtragem considerando apenas os intervalos ao longo do dia em que os retornos são observados. O alisamento das estimativas dos padrões sazonais com o uso de splines cúbicas traz melhorias marginais às estimativas do VaR intradiário. $\mathrm{O}$ método não-paramétrico baseado no quantil empírico, por levar em conta as caudas pesadas da distribuição de retornos intradiários, mostrou-se uma opção a ser considerada. Contudo, o contraste dos resultados obtidos com janelas móveis de diferentes tamanhos inspira cautela na sua utilização e levanta mais uma vez a questão da escolha da janela ótima.

O uso de dados intradiários para a obtenção de previsões da volatilidade diária é factível e apresenta bons resultados. Ambos os métodos avaliados passam no teste de Kupiec em todos os níveis pré-especificados para o VaR, com leve superioridade para o método baseado em alisamento exponencial em relação ao método baseado no cálculo do desvio padrão da amostra com o uso de uma janela móvel de 30 dias. De uma forma geral, o desempenho dos métodos foi melhor que o apresentado pelos métodos tradicionais de estimação de volatilidade diária a partir de retornos diários que avaliamos. Destes, apenas o EWMA com $\lambda=0,94$ apresentou desempenho semelhante.

Cabe ressaltar que os resultados obtidos deixam entrever que os benefícios do uso de dados de alta freqüência no gerenciamento de risco em mercados financeiros podem ser bem maiores. Métodos de filtragem de sazonalidade que considerem um padrão estocástico para a volatilidade, ou mesmo procedimentos determinísticos mais elaborados, bem como modelos de volatilidade que melhor acomodem as características específicas dos dados, podem levar a resultados significativamente melhores no âmbito intradiário. Da mesma forma, os métodos utilizados para estimar a volatilidade diária a partir de retornos intradiários são evidentemente simples. Possivelmente modelos mais sofisticados conseguirão extrair informação relevante das séries históricas de alta freqüência que seja significativamente incre- 
mental àquela fornecida pelas séries diárias, de forma a justificar a maior dificuldade operacional que apresentam. Ainda como sugestão para trabalhos futuros, seria de grande interesse a investigação dos aspectos aqui abordados com relação a outros mercados da América Latina.

\section{Referências}

Andersen, T. G. (2000). Some reflections on analysis of high-frequency data. Journal of Business 83 Economic Statistics, 18(2):146-153.

Andersen, T. G. \& Bollerslev, T. (1997). Intraday periodicity and volatility persistence in financial markets. Journal of Empirical Finance, (4):115-158.

Andersen, T. G. \& Bollerslev, T. (1998a). Answering the skeptics: Yes, standard volatility models do provide accurate forecasts. International Economic Review, 39(4):885-905.

Andersen, T. G. \& Bollerslev, T. (1998b). Deutsche mark-dollar volatility: Intraday activity patterns, macroeconomic announcements, and longer run dependencies. The Journal of Finance, 53(1):219-265.

Andersen, T. G. \& Bollerslev, T. (1999). Forecasting financial market volatility: Sample frequency vis-à-vis forecast horizon. Journal of Empirical Finance, 6:457-477.

Beltratti, A. \& Morana, C. (1999). Computing value at risk with high frequency data. Journal of Empirical Finance, 6:431-455.

Giot, P. (2000a). Intraday value-at-risk. Department of Quantitative Economics, Maastricht University (Holland) and Center for Operations Research and Econometrics (CORE), UCL (Belgium). Disponível em http://www.gloriamundi.org/var/wps.html.

Giot, P. (2000b). Time transformations, intraday data and volatility models. Journal of Computational Finance, 4(2):31-62.

Giot, P. \& Laurent, S. (2001a). Modelling daily value-at-risk using realized volatility and arch type models. Department of Quantitative Economics, Maastricht University (Holland) and Center for Operations Research and Econometrics (CORE), UCL (Belgium) - april/2001. Disponível em http://www.gloriamundi.org/var/wps.html. 
Giot, P. \& Laurent, S. (2001b). Value-at-risk for long and short trading positions. Department of Quantitative Economics, Maastricht University (Holland) and Center for Operations Research and Econometrics (CORE), UCL (Belgium). Disponível em http://www.gloriamundi.org/var/wps.html.

Goodhart, C. A. E. \& O'hara, M. (1997). High frequency data in financial markets: Issues and applications. Journal of Empirical Finance, 4:73-114.

Jorion, P. (1998). Value at risk: A nova fonte de referência para o controle de risco de mercado. Bolsa de Mercadorias e Futuros - São Paulo.

Kupiec, P. (1995). Techniques for verifying the accuracy of risk measurement models. Journal of Derivatives, 2:73-84.

Lemgruber, E. F. \& Cunha, J. (2001). Comparação das metodologias de mapeamento sugeridas pelo RiskMetrics para cálculo de risco de mercado de títulos pré-fixados no Brasil. Publicado em Gestão de Risco e Derivativos: Aplicações no Brasil.

Lemgruber, E. F. \& Ohanian, G. (2001). O modelo de projeção de volatilidade do RiskMetrics e a hipótese de distribuição normal condicional para alguns fatores de risco do Brasil. Publicado em Gestão de Risco e Derivativos: Aplicações no Brasil.

Moreira, J. M. S. (2002). O uso de dados de alta freqüência na estimação de volatilidade e valor em risco para o Ibovespa. Orientador: Eduardo Facó Lemgruber. Rio de Janeiro: UFRJ/COPPEAD, 95 p. Dissertação de Mestrado.

Parkinson, M. (1980). The extreme value method for estimating the variance of the rate of return. Journal of Business, 53(1):61-65.

Riskmetrics Group (1996). RiskMetrics - Technical Document. J. P. Morgan, New York.

Taylor, S. J. \& Xu, X. (1997). The incremental volatility information in one million foreign exchange quotations. Journal of Empirical Finance, 4:317-340. 